\title{
Different routes to pressure-induced volume collapse transitions in gadolinium and terbium metals
}

\author{
G. Fabbris,,${ }^{1,2}$ T. Matsuoka,,${ }^{2,3,4}$ J. Lim, ${ }^{2}$ J. R. L. Mardegan,,${ }^{1,5}$ K. Shimizu, ${ }^{3}$ D. Haskel, ${ }^{1}$ and J. S. Schilling 2 , * \\ ${ }^{1}$ Advanced Photon Source, Argonne National Laboratory, Argonne, IL 60439, USA \\ ${ }^{2}$ Department of Physics, Washington University, St. Louis, MO 63130, USA \\ ${ }^{3}$ KYOKUGEN, Osaka University, 1-3 Machikaneyama, Toyonaka, Osaka 560-8531, Japan \\ ${ }^{4}$ Department of Material Science and Technology, \\ Gifu University, 1-1 Yanagido, Gifu 501-1193, Japan \\ ${ }^{5}$ Instituto de Física Gleb Wataghin, UNICAMP, Campinas, SP 13083-859, Brazil
}

\begin{abstract}
The sudden decrease in molar volume exhibited by most lanthanides under high pressure is often attributed to changes in the degree of localization of their $4 f$-electrons. We give evidence, based on electrical resistivity measurements of dilute $\mathrm{Y}(\mathrm{Gd})$ and $\mathrm{Y}(\mathrm{Tb})$ alloys to $120 \mathrm{GPa}$, that the volume collapse transitions in Gd and $\mathrm{Tb}$ metals have different origins, despite their being neighbors in the periodic table. Remarkably, the change under pressure in the magnetic state of isolated $\operatorname{Pr}$ or Tb impurity ions in the nonmagnetic Y host appears to closely mirror corresponding changes in pure Pr or Tb metals. The collapse in Tb appears to be driven by an enhanced negative exchange interaction between $4 f$ and conduction electrons under pressure (Kondo resonance) which, in the case of $\mathrm{Y}(\mathrm{Tb})$, dramatically alters the superconducting properties of the $\mathrm{Y}$ host, much like previously found for $\mathrm{Y}(\mathrm{Pr})$. In $\mathrm{Gd}$ our resistivity measurements suggest that a Kondo resonance is not the main driver for its volume collapse. X-ray absorption and emission spectroscopies clearly show that $4 f$ local moments remain largely intact across both volume collapse transitions ruling out $4 f$ band formation (delocalization) and valence transition models as possible drivers. The results highlight the richness of behavior behind the volume collapse transition in lanthanides and demonstrate the stability of the $4 f$ level against band formation to extreme pressure.
\end{abstract}

\section{INTRODUCTION}

One of the central questions in the magnetism of solids is whether the electrons responsible for the magnetic phenomena are localized or itinerant in nature. This dual character emerges in actinides, where the $5 f$ level is close to a localized-itinerant boundary, leading to a large diversity of physical properties and crystal structures $\stackrel{1}{=}$ In lanthanides the $4 f$ level is atomic-like at ambient pressure so that its contribution to the material properties only occurs through interaction with the conduction electrons. Despite the significant amount of work devoted to $4 f$ and $5 f$ electron systems over many years, the theoretical treatment of these levels is still very challenging. Recent advances in dynamical mean field theory have been encouraging, $\stackrel{2-6}{\underline{6}}$ but the agreement with experiment is still incomplete. In analogy to actinides, the localized character of the lanthanide $4 f$ level is expected to change under sufficient pressure ${ }^{7}$ In particular, a sudden pressure-induced drop in the molar volume, commonly termed "volume collapse", has been observed in Ce (16\%

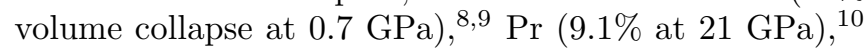

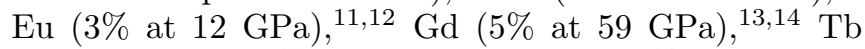
$(5 \%$ at $53 \mathrm{GPa}) \stackrel{15}{=} \mathrm{Dy}(6 \%$ at $73 \mathrm{GPa}) \stackrel{16}{\stackrel{16}{r} \mathrm{Ho}}(3 \%$ at $103 \mathrm{GPa}) \stackrel{17}{,} \mathrm{Tm}(1.5 \%$ at $120 \mathrm{GPa}), \frac{18}{,}$ and $\mathrm{Lu}(5 \%$ at $90 \mathrm{GPa}) \stackrel{19}{\underline{3}}$ Such volume collapses are still the subject of debate,,$\frac{3,5,6,20-25}{2}$ although widely thought to result from changes in the degree of $4 f$ localization.

Here we focus on the volume collapse phenomena because the sudden and often sizeable change in the molar volume, and accompanying changes in electronic and magnetic properties, should facilitate the identification of its origin. In addition, except for Ce, the volume collapse is accompanied by a transition to a lower symmetry crystal structure also found in light actinides with itinerant $5 f$ electrons, suggesting that a fundamental change in the character of the $4 f$ electrons, perhaps from local to itinerant, may take place.

Three models often invoked to describe pressureinduced volume-collapse phenomena in the lanthanides are: (1) valence transition model,$\underline{26}$ where a $4 f$ electron is transferred into the $s p d$-electron conduction band causing a sudden reduction in the ionic radius and enhanced metallic binding; (2) Mott-Hubbard model, $\underline{\underline{7}}$ where the $4 f$ states undergo a local-to-itinerant transition, the $4 f$ electrons making a significant contribution to crystalline binding; and, (3) Kondo volume collapse model, 27 where the approach of the localized $4 f$ level to the Fermi energy under pressure leads to a sharp increase in the Kondo temperature $T_{\mathrm{K}}$. In all three models the $4 f$ electrons play a critical role.

It is important to note, however, that 4 -electron involvement is not required for a volume collapse to occur. The transition metal elements $\mathrm{Y}$ and Sc lack $4 f$ electrons, but display volume collapses of $3 \%$ (at $99 \mathrm{GPa}^{28}$ ) and $4 \%$ (at $140 \mathrm{GPa}^{29}$ ), respectively; both are trivalent with conduction electrons whose $s p d$-character closely matches that of the trivalent lanthanides. In fact, a volume collapse is observed in many elements and compounds devoid of $4 f$ electrons ${ }^{30-33}$ The volume collapse in $\mathrm{Y}$ and $\mathrm{Sc}$ is likely promoted by the ubiquitous $s \rightarrow d$ charge transfer under pressure whereby the number of $d$ electrons in the conduction band $n_{d}$ increases. In fact, across the entire lanthanide series it has been shown that the variation 
in $n_{d}$ plays the dominant role in determining the crystal structure at both ambient and high pressure 34 For this reason it is important to realize that simple $s \rightarrow d$ charge transfer must also be considered as a viable model for pressure-induced volume collapse in all lanthanides, whether they contain $4 f$ electrons or not.

The isostructural $\gamma \rightarrow \alpha$ phase transition in Ce at $0.7 \mathrm{GPa}$ exhibits the largest (16\%), and most thoroughly studied, volume collapse of any lanthanide $\stackrel{\underline{8} \cdot 9}{ }$ That the $4 f$ electrons play an important role in this transition is clear from the large $(80 \%)$ and abrupt drop in the magnetic susceptibility at $0.7 \mathrm{GPa}, \stackrel{35}{=}$ thus supporting the Kondo or Mott-Hubbard models. However, recent results also point to the importance of lattice dynamics in Ce's volume collapse. $\underline{\underline{24}}$

For Gd and the heavier lanthanides, relatively high pressures (> $>0 \mathrm{GPa}$ ) are required to trigger the volume collapse. Due to the technical challenges associated with experiments at these higher pressures, the volume collapse in the heavier lanthanides has received less attention. Both Gd and $\mathrm{Tb}$ display a $5 \%$ volume collapse at, respectively, $59 \mathrm{GPa}$ and $53 \mathrm{GPa}$ to a monoclinic $C 2 / \mathrm{m}$ structure $\stackrel{13,15}{=}$ The emergence of such a low-symmetry phase typical for the light actinides is usually attributed to the onset of $4 f$ binding, i.e. the Mott-Hubbard picture. However, the same phase is found in pure Y near 1 Mbar, 28 thus an $s \rightarrow d$ transfer scenario cannot be ruled out. Both theoretical ${ }^{36}$ and $\mathrm{x}$-ray spectroscopic 21,23 studies report that Gd's bare local moment remains intact through the volume collapse transition. This result is consistent with both the Kondo collapse picture or simple $s \rightarrow d$ charge transfer. A continuous increase in hybridization between $4 f$ and conduction electrons under pressure was observed in resonant inelastic x-ray scattering (RIXS) measurements for Gd and interpreted as evidence for a Kondo driven volume collapse model ${ }^{21}$ However, no clear correlation between the degree of $4 f$ delocalization and the volume collapse transition in $\mathrm{Gd}$ could be established. Therefore, the mechanism responsible for the volume collapse in $\mathrm{Gd}, \mathrm{Tb}$, and the remaining heavy lanthanides remains unclear.

We examine the origin of the pressure-induced volume collapse in Gd and $\mathrm{Tb}$ by carrying out electrical resistivity measurements on dilute $\mathrm{Y}(\mathrm{Gd})$ and $\mathrm{Y}(\mathrm{Tb})$ magnetic alloys to pressures as high as $120 \mathrm{GPa}$. The suppression of superconductivity in the $\mathrm{Y}$ host is used to probe changes in the magnetic state of the Tb and Gd ions, e.g., as a result of Kondo screening. Since the spd-character of conduction electrons in $\mathrm{Y}$ closely matches that of the trivalent lanthanides, experiments on these diluted $\mathrm{Y}$ alloys are expected to mimic the interaction between $4 f$ and conduction electrons in the pure $\mathrm{Tb}$ and Gd metals, provided that $4 f-4 f$ overlap is minimal. X-ray absorption near edge structure (XANES) and non-resonant x-ray emission spectroscopy (XES) measurements carried out in $\mathrm{Tb}$ metal under pressure in this work, together with similar measurements already published for $\mathrm{Gd} \stackrel{21,23}{\stackrel{2}{2}}$ indeed corroborate that direct $4 f-4 f$ interac- tions remain largely unchanged in the studied pressure range, as seen from the absence of changes in local moment. The XANES and XES measurements allow us to probe $s \rightarrow d$ charge transfer and possible changes in $4 f$ valence and local moments. For Tb our spectroscopic results exclude the valence transition and Mott-Hubbard scenarios and provide evidence that a pressure-induced $s \rightarrow d$ charge transfer takes place. However, the electrical resistivity measurements strongly suggest that the volume collapse in $\mathrm{Tb}$ has a significant magnetic component and is, in fact, triggered by the many-body Kondo resonance. For Gd, on the other hand, we do not observe any clear signature of a Kondo resonance in the $\mathrm{Y}(\mathrm{Gd})$ alloys in the vicinity of the volume collapse transition. Considering that such signatures are clearly observed in $\mathrm{Y}(\mathrm{Pr})$ and $\mathrm{Y}(\mathrm{Tb})$ alloys in the vicinity of their volume collapses, we conclude that a Kondo-driven collapse in Gd is unlikely. Taken together with previous x-ray spectroscopic results on Gd showing absence of $4 f$ band formation or loss of local moments but significant $s \rightarrow d$ charge-transfer, we suggest that the latter may be the driving force behind the volume collapse in Gd metal.

\section{EXPERIMENTAL}

Dilute magnetic alloys $\mathrm{Y}(0.5$ at.\% $\mathrm{Tb}), \mathrm{Y}(0.5$ at.\% $\mathrm{Gd})$, and $\mathrm{Y}(1$ at.\% Pr) were prepared by argon arcmelting stoichiometric amounts of $\mathrm{Y}$ and dopant (Tb, $\mathrm{Pr}$, Y - 99.9\% pure, Material Preparation Center of the Ames Laboratory; ${ }^{37}$ Gd - 99.9\% pure, Alfa Aesar). The melting procedure was repeated several times to promote homogeneity, the weight loss being always less than $0.1 \%$ of total mass. No significant concentration of other impurities or clustering was detected by x-ray fluorescence (XRF) and x-ray absorption fine structure (XAFS) measurements, as detailed in the next section. The high-pressure DC electrical resistivity measurements were performed in a membrane-driven diamond-anvil cell with both standard (300 $\mu \mathrm{m}$ culet diameter) and beveled (350 to $180 \mu \mathrm{m}$ culet diameter) anvils. A rhenium gasket was insulated using a 4:1 c-BN-epoxy mixture which also served as pressure medium. The ruby fluorescence technique was used to determine pressure in all experiments $\stackrel{38}{=}$ Four-point resistance was measured using leads cut from a thin $\mathrm{Pt}$ foil. The current used was chosen to keep the power dissipated in the sample always below $0.5 \mu \mathrm{W}$. The pressure cell was cooled using an Oxford He flow cryostat; after the initial cooling, the temperature was always kept below $120 \mathrm{~K}$. The sample's lateral dimension was $\sim 1 / 3$ of culet diameter; the thickness was $<20 \mu \mathrm{m}$. The small sample was placed on top of the Pt leads and electrical contact was made by pressing the sample into the leads. Further experimental details are given elsewhere $\underline{39}$

$\mathrm{X}$-ray absorption near edge structure (XANES) measurements were carried out on a $\mathrm{Tb}$ foil at the $\mathrm{L}_{3}$ absorption edge $(2 p \rightarrow 5 d$ transition) at PNC/XSD (20BM) beamline of the Advanced Photon Source (APS), 
Argonne National Laboratory (ANL). A "symmetric" cell (Princeton University) was prepared with diamonds of $300 \mu \mathrm{m}$ bevelled to $180 \mu \mathrm{m}$ culet diameter. A partially perforated plus full anvil pair was used to reduce anvil attenuation of x-ray intensity and improve counting statistics. A rhenium gasket was pre-indented to $30 \mu \mathrm{m}$, and a sample chamber of $80 \mu \mathrm{m}$ diameter was laser drilled in the center of the indentation. A small piece of Tb foil (Alfa Aesar, 99.9\% purity) was loaded together with ruby balls, the later used for pressure calibration. Neon pressure medium was loaded using the COMPRESS/GSECARS system. $\underline{\underline{40}}$ The experiment was performed at room temperature, and pressure applied manually using the cell screws. XANES was measured in transmission mode, both incident and transmitted intensities were detected using $\mathrm{N}_{2}$ filled ion chambers. KirkpatrickBaez mirrors focused the x-ray beam to $\sim 3 \mathrm{x} 5 \mu \mathrm{m}^{2}$.

Non-resonant $\mathrm{Tb} \mathrm{L}_{\gamma} \mathrm{x}$-ray emission (XES) experiments were performed at HP-CAT (16-ID-D) beamline of the APS, ANL. A "symmetric" cell was used with full diamonds of $300 \mu \mathrm{m}$ culet diameter. Photon energy was fixed at $11.3 \mathrm{keV}$. Data were collected using the diamondin gasket-out geometry, thus an x-ray transparent Be gasket, pre-indented to $50 \mu \mathrm{m}$, was used. The center of the gasket was replaced with a pressed c-BN/Epoxy insert. A $100 \mu \mathrm{m}$ hole was carefully laser drilled in the center of the insert, and used as sample chamber. The cell was loaded with Tb foil (Alfa Aesar, 99.9\% purity) and ruby balls; Si oil was used as pressure media. The experiment was performed at room temperature, and pressure applied manually using the cell screws. XES data were measured using a scintillator detector coupled to a $\mathrm{Si}$ (444) analyzer. The data were normalized by the incident beam intensity which was detected with a $\mathrm{N}_{2}$ filled ion chamber. Kirkpatrick-Baez mirrors focused the x-ray beam to $\sim 40 \times 60 \mu \mathrm{m}^{2}$.

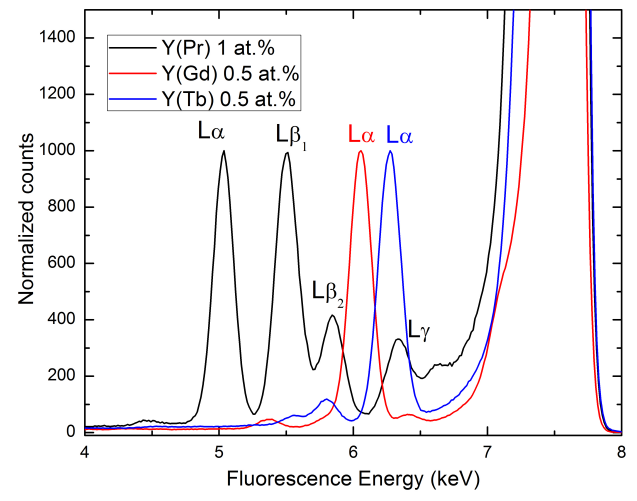

FIG. 1. (color online) X-ray fluorescence spectra. Data was taken at an incidence energy of $7.55 \mathrm{keV}$ and normalized to the $\mathrm{L}_{\alpha}$ peak intensity.

\section{SAMPLE CHARACTERIZATION}

XRF measurements were carried out on the three $\mathrm{Y}(\mathrm{RE})(\mathrm{RE}=\mathrm{Pr}, \mathrm{Gd}, \mathrm{Tb})$ alloys in order to verify the dopant content and check for unwanted impurities. The measurements were performed at the 4-ID-D beamline of the APS, ANL. An incident photon energy of $7.55 \mathrm{keV}$ was used and fluorescence photons were collected using a four-element silicon drift energy dispersive detector in a normal incidence geometry. Measurements were performed at room temperature. Fig. 1 shows the data normalized so that the $\mathrm{L}_{\alpha}$ peak has 1000 counts. The incident photon energy lies between the $\mathrm{L}_{3}$ and $\mathrm{L}_{2}$ edges for $\mathrm{Gd}$ and $\mathrm{Tb}$, but above $\mathrm{L}_{2}$ for $\mathrm{Pr}$, thus extra fluorescence lines appear in the latter's spectrum. This result confirm the presence of dopants and absence of significant presence of other impurities.

Despite the similar ionic radii of $\mathrm{Y}$ and dopants, the absence of dopant clustering has to be verified as such clustering could lead to magnetically ordered islands inside the Y host and potentially affect the value of superconducting transition temperatures, $T_{\mathrm{c}}$, in the $\mathrm{Y}$ alloys. XAFS measurements were carried out to probe the local environment around dopants. Experiments were performed at APS beamline 4-ID-D at the $\mathrm{L}_{3}$ edge of $\mathrm{Pr}$

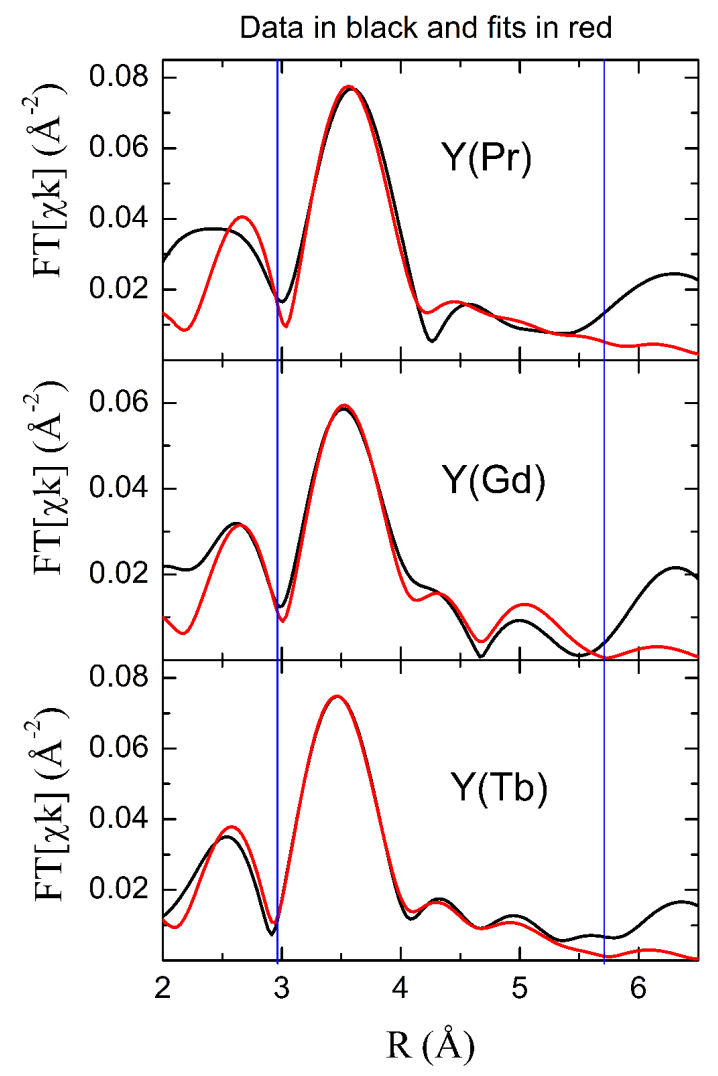

FIG. 2. (color online) Fourier transform of the XAFS data collected at the $\mathrm{L}_{3}$ edge of $\mathrm{Pr}, \mathrm{Gd}$ and $\mathrm{Tb}$ in their respective alloys 
TABLE I. Y-RE measured here compared to RE-RE distances obtained by diffraction. ${ }^{44} \mathrm{Y}-\mathrm{Y}$ distance: $3.6474(7) \AA .44$

\begin{tabular}{ccc}
\hline \hline $\mathrm{RE}$ & XAFS $(\AA)$ & Diffraction $(\AA)$ \\
\hline $\mathrm{Pr}$ & $3.65(2)$ & $3.6725(7)$ \\
$\mathrm{Gd}$ & $3.62(2)$ & $3.6360(9)$ \\
$\mathrm{Tb}$ & $3.59(1)$ & $3.6010(3)$ \\
\hline \hline
\end{tabular}

(5.964 keV), Gd (7.243 keV), and Tb (7.514 keV) using the same fluorescence geometry as in the XRF measurements. The photoelectron wavenumber was limited by the presence of the $\mathrm{L}_{2}$ edge. All experiments were performed at room temperature. XAFS data were analysed using IFEFFIT/HORAE ${ }^{41,42}$ and FEFF6 ${ }^{43}$ software. The spectra in the $\mathrm{k}=3-8 \AA^{-1}$ range were truncated using a Hanning window and fits were perfomed in real space within $\mathrm{r}=2.8-5.3 \AA$ up to the third coordination shell. XAFS spectra and fits are shown in Fig. 2. Fits with mixtures of RE-Y and RE-RE first neighbor distances were attempted. For the three samples the fraction of RE-RE neighbors was zero within experimental error $(\sim 5 \%)$, proving that no significant RE clustering is present. XAFS shows a systematic reduction in Y-RE distance in going from $\mathrm{Pr}$ to $\mathrm{Tb}$ (see Table I) as expected from the well known lanthanide contraction. Furthermore, the Y-RE distances are in very close agreement with the RE-RE distances in the pure compounds (Table If we note that the first neighbor distances for the pure $\mathrm{Y}$ and RE metals are similar $\left.{ }^{44}\right)$. The presence of a clear lanthanide contraction in the $\mathrm{Y}(\mathrm{RE})$ alloys, which results from the response of outer $s p d$ valence electrons to an increasingly attractive nuclear potential poorly screened by the additional $4 f$ electrons, is additional evidence for the closely matched character of spd conduction electrons in the $\mathrm{Y}(\mathrm{RE})$ alloys and the pure RE metals. This in turn provides sensible justification for mapping results on the interaction between conduction electrons and local moments obtained from electrical resistivity measurements in the $\mathrm{Y}(\mathrm{RE})$ alloys to their pure RE metal counterparts.

\section{RESULTS AND DISCUSSION}

Before considering our present experimental findings on $\mathrm{Gd}$ and $\mathrm{Tb}$, it is useful to first discuss earlier experiments on Ce and Pr. An almost forgotten strategy to test for the presence of Kondo effect phenomena is to alloy a very dilute concentration of the magnetic component into a superconducting host and see whether the pressure dependence of $T_{\mathrm{c}}$ suffers a characteristic "sinkholelike" suppression. $\stackrel{45}{ }$ Maple, Wittig and Kim ${ }^{46}$ carried out such experiments on dilute magnetic alloys of $\mathrm{La}(\mathrm{Ce})$ and found that $T_{\mathrm{c}}$ shows a dramatic "Kondo-sinkhole" suppression around $0.7 \mathrm{GPa}$, close to the pressure where the volume collapse in pure Ce occurs. The effect of the very strong pair-breaking associated with the Kondo effect in dilute magnetic alloys has received considerable theoretical support $47-50$ which has aided in the understanding of such complex and interesting behavior as the reentrant superconductivity observed in $\mathrm{La}(\mathrm{Ce}) \mathrm{Al}_{2} \cdot 51$

As a second example consider $\mathrm{Pr}$, which suffers a $10 \%$ volume collapse at $21 \mathrm{GPa} . \stackrel{10,54}{4}$ As for $\mathrm{La}(\mathrm{Ce}) \stackrel{46}{,}$ a marked suppression of $T_{\mathrm{c}}$ is observed in the dilute magnetic alloys $\mathrm{La}(\mathrm{Pr})^{53}$ and $\mathrm{Y}(\mathrm{Pr})^{55}$ beginning near $21 \mathrm{GPa}$,
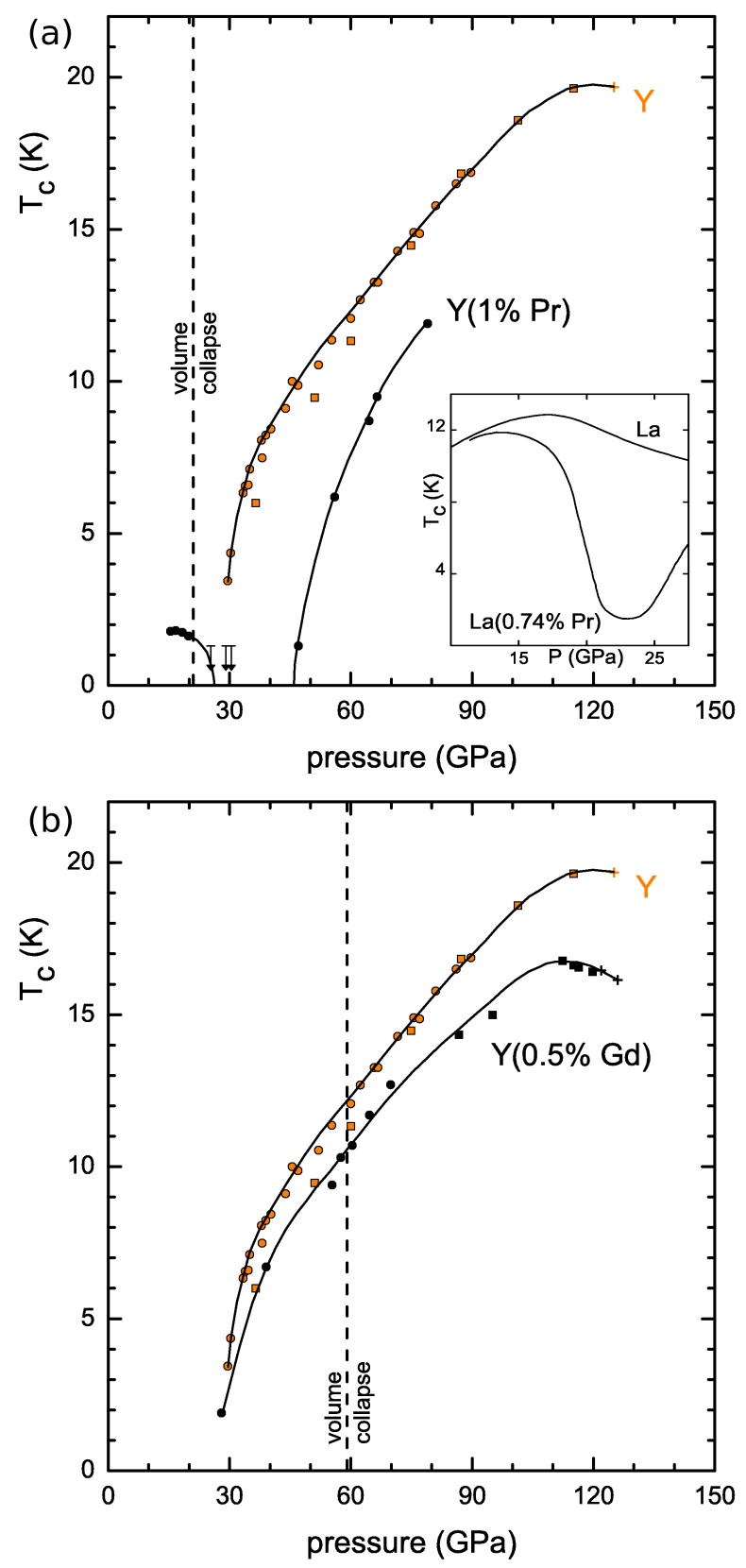

FIG. 3. (color online) Pressure dependence of $T_{\mathrm{c}}$ for (a) $\mathrm{Y}(1$ at.\% Pr) and (b) Y $\left(0.5\right.$ at.\% Gd) compared to that for $\mathrm{Y}^{52}$ Vertical dashed lines show the critical pressure for the volume collapse in $\mathrm{Pr}$ and $\mathrm{Gd}$. Inset in (a) shows data for La and $\mathrm{La}(0.74$ at.\% Pr) adapted from Fig. 2 of Ref. 53 . 
the pressure where Pr's volume collapse occurs. These experiments were limited to $27 \mathrm{GPa}$ so the full recovery of $T_{\mathrm{c}}(\mathrm{P})$ was not observed (see inset to Fig. 3(a)). Fig. 3(a) displays our recent measurements on $\mathrm{Y}(1$ at.\% Pr $)$ which extend the previous work to much higher pressures. For pressures well above $40 \mathrm{GPa}, T_{\mathrm{c}}(\mathrm{P})$ again approaches that for pure Y. Exactly this behavior is expected as the Kondo temperature $T_{\mathrm{K}}$ is rapidly pushed under pressure to values far above $T_{\mathrm{c}}$ where pair-breaking significantly weakens and the spin-compensated magnetic impurity appears nonmagnetic to the Y host. $\underline{\underline{47}} \underline{\underline{50}}$ XANES studies confirmed that $\mathrm{Pr}$ remains trivalent to $26 \mathrm{GPa}, \underline{56}$ XES studies on Pr metal also find no change in the bare local magnetic moment across the volume collapse transition,, 22 and evidence for $4 f$-conduction elec-

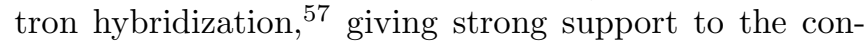
clusion that this transition in $\mathrm{Pr}$ is Kondo-driven.

We now turn to Gd. Since the $4 f^{7}$ orbital in Gd metal is half filled, its local magnetic state is the most stable of all lanthanides; Gd's $4 f^{7}$ level, in fact, is located $\sim 9 \mathrm{eV}$ below the Fermi level ${ }^{36}$ XES experiments show no change in the local magnetic moment of Gd across the volume collapse transition ${ }^{21,23}$ which excludes the if local-itinerant (Mott-Hubbard) transition model. A small increase in the degree of $4 f^{8}$ character observed in resonant $\mathrm{L}_{\alpha}$ XES experiments ${ }^{21}$ was interpreted as possible evidence for Kondo-like behavior in Gd. However, no correlation was found between the extent of $4 f$ conduction electron hybridization under pressure and the occurrence of the volume collapse transition. Such increase in hybridization at high pressure may be interpreted as a small valence increase; however, no valence changes were observed with XANES, $\underline{23}$ indicating that Gd remains close to $3+$ across the volume collapse, and excluding the valence transition model.

Further insight into the mechanism behind the volume collapse transition in $\mathrm{Gd}$ is given by the $T_{\mathrm{c}}(\mathrm{P})$ data shown in Fig. 3(b) for pure $\mathrm{Y}$ and $\mathrm{Y}(0.5$ at.\% Gd). Compared to La, Y is a superior superconducting host for the present studies since its ionic radius closely matches that of the heavy lanthanides and, above $20 \mathrm{GPa}, T_{\mathrm{c}}$ increases in a simple, monotonic manner to pressures as high as $120 \mathrm{GPa}$ (see Fig. (3), $\frac{52}{5}$ In contrast to what is observed for $\mathrm{La}(\mathrm{Ce}), \mathrm{La}(\mathrm{Pr})$, and $\mathrm{Y}(\mathrm{Pr})$ alloys, no "Kondosinkhole" or marked suppression of $T_{\mathrm{c}}(\mathrm{P})$ is observed in $\mathrm{Y}(\mathrm{Gd})$ at a pressure anywhere near that (59 GPa) where Gd's volume collapse occurs. This result suggests that the volume collapse in Gd is neither due to the giant Kondo resonance nor is magnetic in origin. We note that this is not inconsistent with RIXS results 21 which show a continuous increase in hybridization between $4 f$ and conduction electrons under pressure but do not establish a correlation between this increase and the volume collapse transition in Gd. Since robust local moments remain present at the collapse transition in Gd, ruling out

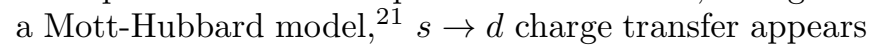
to be the dominant driving force for the volume collapse in Gd. This charge transfer causes a strong reduction in the area of the main absorption peak in Gd $\mathrm{L}_{3}$ XANES data with pressure 23

$\mathrm{Tb}$ is much more likely than Gd to exhibit $4 f$-driven instabilities under pressure, since Tb's $4 f^{8}$ level lies only $\sim 3 \mathrm{eV}$ below the Fermi energy ${ }^{58}$ To establish if the Kondo resonance plays a role in Tb's volume collapse, we carried out high-pressure resistivity studies on $\mathrm{Y}(0.5$ at.\% $\mathrm{Tb})$. The $T_{\mathrm{c}}(\mathrm{P})$ is plotted in Fig. 4 for three independent runs and compared to that for pure Y. Beginning at about $53 \mathrm{GPa}$, a marked suppression of $T_{\mathrm{c}}$ is evident for the alloy with increasing pressure. Within experimental error, this onset pressure closely matches that (53 GPa) where the volume collapse occurs in Tb. The width of the resistive transition at $30.9 \mathrm{GPa}$ (see inset to Fig. 2) arises from the pressure gradient across the sample. That this width becomes very narrow at $81.4 \mathrm{GPa}$ is consistent with the fact that for pressures above $50 \mathrm{GPa}$ $T_{\mathrm{c}}$ is constant. The present experiments thus suggest that the Kondo effect plays a role in the volume collapse transition of $\mathrm{Tb}$ metal.

High-pressure $\mathrm{L}_{3}$ XANES data on $\mathrm{Tb}$ are presented in Fig. [5(a). Were Tb to undergo a $4 f^{8}$ to $4 f^{7}$ valence transition under pressure, a peak would appear at the position of the arrow under 4+; no such transition is observed to $65 \mathrm{GPa}$. Thus a valence transition does not contribute to the volume collapse in $\mathrm{Tb}$ at $53 \mathrm{GPa}$. In Fig. [5(a) it is clearly seen that the $3+$ absorption peak is reduced in area with pressure. The $\mathrm{L}_{3}$ absorption edge is dominated by the dipolar $2 p_{3 / 2} \rightarrow 5 d$ electronic exci-

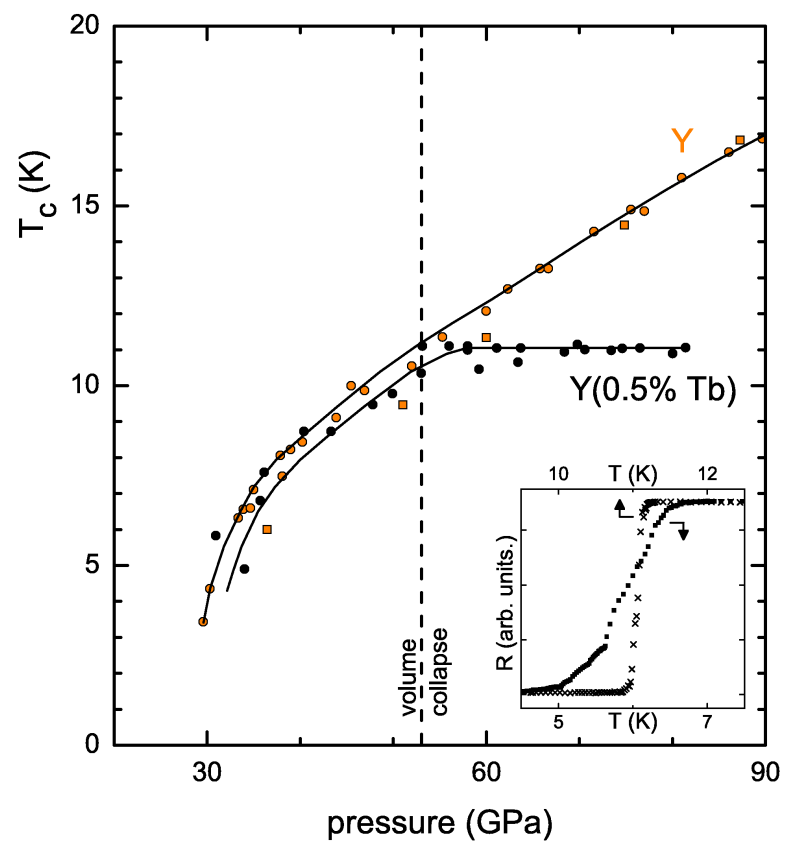

FIG. 4. (color online) Pressure dependence of $T_{\mathrm{c}}$ for $\mathrm{Y}(0.5$ at.\% $\mathrm{Tb}$ ) alloy. Vertical dashed line marks pressure (53 GPa) of volume collapse in $\mathrm{Tb}$. Inset shows resistive superconducting transition at $81.4 \mathrm{GPa}(\times)$ is much narrower than that at 30.9 GPa (ם). 
tation; thus the area of the absorption peak is directly related to the number of empty $5 d$ states. These results indicate that $s \rightarrow d$ charge transfer does indeed occur in $\mathrm{Tb}$ under pressure, suggesting that this mechanism may also contributes to Tb's volume collapse. However, while $s \rightarrow d$ transfer occurs throughout the entire pres-

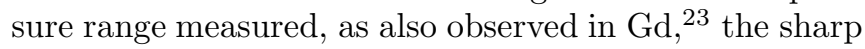
deviation in $T_{c}(\mathrm{P})$ due to strong Kondo pair breaking begins at the pressure (53 GPa) where the volume collapse occurs in $\mathrm{Tb}$, much like what is found for Ce and Pr. The Kondo resonance thus appears to be the main driver in Tb's volume collapse.

Changes in the character of Tb's $4 f^{8}$ local magnetic moment can be studied using XES. The XES $\mathrm{L}_{\gamma}$ line is shown in Fig. 5(b) at various pressures up to $70 \mathrm{GPa}$. In this experiment a $2 p_{1 / 2}$ electron is excited using highenergy x-ray photons. The hole is then filled by a $4 d_{3 / 2}$ electron, and the energy of the resulting $\mathrm{L}_{\gamma} \mathrm{x}$-ray emission is analyzed with an x-ray spectrometer (Fig. 5(b)). The probability of the $4 d_{3 / 2} \rightarrow 2 p_{1 / 2}$ transition depends
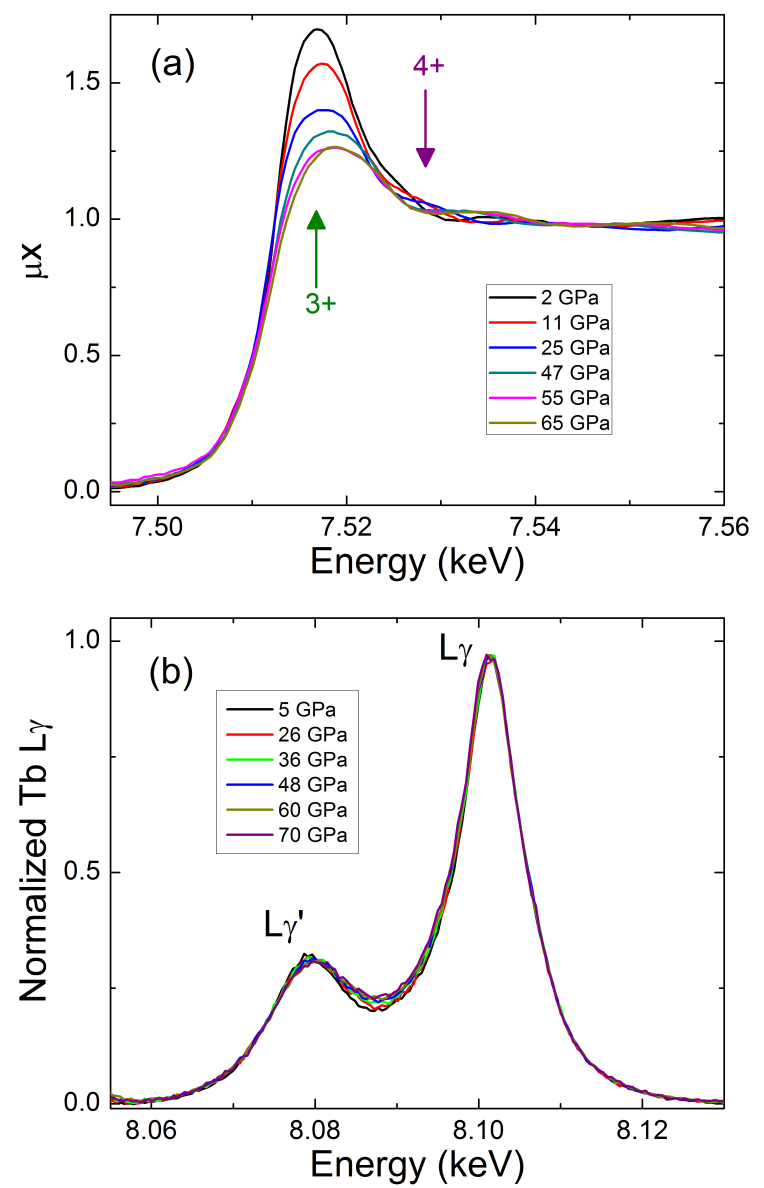

FIG. 5. (color online) (a) Pressure dependence of $\mathrm{L}_{3}$ XANES for Tb. No $4+$ or mixed valence state is observed. Pressureinduced reduction of peak height is direct measure of $\mathrm{s} \rightarrow \mathrm{d}$ transfer. (b) Pressure dependence of $\mathrm{L}_{\gamma}$ non-resonant XES for Tb. A $4 f^{8}$ local-itinerant transition is not observed. on the initial $\left(2 p_{1 / 2}^{1} 4 d_{3 / 2}^{4}\right)$ and final $\left(2 p_{1 / 2}^{2} 4 d_{3 / 2}^{3}\right)$ states. The final $4 d_{3 / 2}^{3}$ state is split by the exchange interaction with the $4 f^{8}$ level which leads to the splitting of the $\mathrm{L}_{\gamma}$ line seen in Fig. 5(b). The ratio between the peak intensities is known to be related to the total angular momentum of the $4 f$ state $\frac{\sqrt[5]{21}, 59}{50}$ significant change in the spectrum is observed throughout the pressure range measured, and, in particular, no discontinuous change in the data is observed when the collapsed phase is reached. The near constancy of the $\mathrm{L}_{\gamma}$ splitting gives direct evidence that the local character of the $4 f^{8}$ orbital is preserved up to $75 \mathrm{GPa}$, thus excluding the possibility that the Mott-Hubbard ( $f f$ local-itinerant) mechanism contributes to the volume collapse in $\mathrm{Tb}$.

\section{SUMMARY}

In conclusion, taken together, the present resistivity and $\mathrm{x}$-ray spectroscopy studies give evidence that the volume collapse in $\mathrm{Tb}$ metal at $53 \mathrm{GPa}$ arises predominantly from the Kondo many-body resonance, a conclusion we reached earlier for Pr. Furthermore, we infer that the volume collapse in Gd is unlikely to be Kondo-driven, a conclusion based on the absence of any measurable effect on the $T_{c}$ of $\mathrm{Y}(\mathrm{Gd})$ alloys anywhere near the volume collapse transition. We postulate that simple $s \rightarrow d$ charge transfer is the main driver for the volume collapse in $\mathrm{Gd}$, thus emphasizing the importance of considering this model as a viable explanation for volume collapse phenomena in $4 f$ and $5 f$ electron systems.

It may seem remarkable that the changes in the magnetic properties under pressure of very dilute $\mathrm{Pr}$ or $\mathrm{Tb}$ impurity ions in a nonmagnetic, superconducting host (Y) so closely parallel the corresponding changes in pure $\mathrm{Pr}$ or $\mathrm{Tb}$ metal, as evidenced by the volume collapse. That this is no accident is corroborated by very recent experiments on $\mathrm{Y}(\mathrm{Dy})$ and Dy ${ }^{60}$ As remarked earlier, the closely matched character of $s p d$ conduction electrons in the $\mathrm{Y}(\mathrm{RE})$ alloys and the pure RE metals provide a natural explanation for this behavior. It appears that the similarity in the interaction between these conduction electrons and local $4 f$ moments in the $\mathrm{Y}(\mathrm{RE})$ alloys and the pure RE metal counterparts, together with the absence of significant $4 f$ bonding, allow for mapping the observations from electrical resistivity measurements in $\mathrm{Y}(\mathrm{RE})$ alloys to their RE metal counterparts. The lanthanide contraction observed by XAFS in the Y(RE) alloys, which mimics the contraction observed in the pure $\mathrm{RE}$ metals, provides further validation for the correspondence in the electronic structure of outer valence electrons in the dilute RE alloys and RE metals.

The $5 f$ level in actinides is known to be close to a local-itinerant transition, $\stackrel{1}{=}$ which has been expected to emerge in the lanthanide $4 f$ electrons by $\sim 1$ Mbar within the Mott-Hubbard model $\underline{\underline{7}}$ However, the present results together with previous x-ray spectroscopy work at high pressures indicate that the lanthanide $4 f$ level is consid- 
erably more stable than what is oftentimes assumed. For all lanthanides, except possibly Ce, pressures well beyond 1 Mbar appear to be required to render the $4 f$ electrons itinerant. This is consistent with suggestions of Yin and Pickett for $\mathrm{Gd}^{36}$ and also with considerations based on the degree of nearest-neighbor $4 f$ orbital overlap for all lanthanides. 61

\section{ACKNOWLEDGMENTS}

Research at both Washington University and the APS was supported by the National Science Foundation through grant DMR-1104742 and by the Carnegie/DOE
Alliance Center (CDAC) through NNSA/DOE Grant No. DE-FC52-08NA28554. Work at Argonne is supported by the US Department of Energy (DOE), Office of Science, Office of Basic Energy Sciences, under Contract No. DEAC-02-06CH11357. J. R. L. Mardegan was supported by FAPESP (SP-Brazil) under contract No. 2011/24166-0. The authors would like to thank Anup Gangopadhyay for assistance in sample preparation, as well as Börje Johansson and James Hamlin for critically reading the manuscript. We thank Steve Heald and Chengjun Sun for their support at Advanced Photon Source (APS) 20BM, Yuming Xiao, Paul Chow and Genevieve Boman for their support at APS HPCAT 16-ID-D, and Sergey Tkachev for his help in using the APS GSECARS gas loading system.
* Corresponding author: jss@wuphys.wustl.edu

1 K. Moore and G. van der Laan, Rev. Mod. Phys. 81, 235 (2009).

2 M. B. Zölfl, I. A. Nekrasov, T. Pruschke, V. I. Anisimov, and J. Keller, Phys. Rev. Lett. 87, 276403 (2001).

3 A. K. McMahan, K. Held, and R. T. Scalettar, Phys. Rev. B 67, 075108 (2003).

4 A. McMahan, Phys. Rev. B 72, 115125 (2005).

${ }^{5}$ M. Lipp, A. Sorini, J. Bradley, B. Maddox, K. Moore, H. Cynn, T. Devereaux, Y. Xiao, P. Chow, and W. Evans, Phys. Rev. Lett. 109, 195705 (2012).

6 N. Lanatà, Y.-X. Yao, C.-Z. Wang, K.-M. Ho, J. Schmalian, K. Haule, and G. Kotliar, Physical Review Letters 111, 196801 (2013).

7 B. Johansson, Philos. Mag. 30, 469 (1974).

8 A. Lawson and T.-Y. Tang, Phys. Rev. 76, 301 (1949).

9 M. Lipp, D. Jackson, H. Cynn, C. Aracne, W. Evans, and A. McMahan, Phys. Rev. Lett. 101, 165703 (2008).

10 N. Cunningham, N. Velisavljevic, and Y. Vohra, Phys. Rev. B 71, 012108 (2005).

11 K. Takemura and K. Syassen, J. Phys. F: Metal Phys. 15, 543 (1985).

12 W. Bi, Y. Meng, R. S. Kumar, A. L. Cornelius, W. W. Tipton, R. G. Hennig, Y. Zhang, C. Chen, and J. S. Schilling, Phys. Rev. B 83, 104106 (2011).

${ }^{13}$ H. Hua, V. K. Vohra, J. Akella, S. T. Weir, R. Ahuja, and B. Johansson, Rev. High Pressure Sci. Technol. 7, 233 (1998).

14 D. Errandonea, R. Boehler, B. Schwager, and M. Mezouar, Phys. Rev. B 75, 014103 (2007).

15 N. Cunningham, W. Qiu, K. Hope, H.-P. Liermann, and Y. Vohra, Phys. Rev. B 76, 212101 (2007).

16 R. Patterson, J. Appl. Phys. 95, 5443 (2004).

17 Y. K. Vohra, B. R. Sangala, A. K. Stemshorn, and K. M. Hope, Mater. Res. Soc. Symp. Proc. 1104, NN01 (2008).

18 J. M. Montgomery, G. K. Samudrala, G. M. Tsoi, and Y. K. Vohra, J. Phys.: Condens. Matter 23, 155701 (2011).

19 G. Chesnut and Y. Vohra, Phys. Rev. B 57, 10221 (1998).

20 J.-P. Rueff, J.-P. Itié, M. Taguchi, C. Hague, J.-M. Mariot, R. Delaunay, J.-P. Kappler, and N. Jaouen, Phys. Rev. Lett. 96, 237403 (2006).

21 B. Maddox, A. Lazicki, C. Yoo, V. Iota, M. Chen,
A. McMahan, M. Hu, P. Chow, R. Scalettar, and W. Pickett, Phys. Rev. Lett. 96, 215701 (2006).

22 B. Maddox, Pressure-Induced Electronic Phase Transitions in Transition Metal Oxides and Rare Earth Metals, Ph.d. thesis, University of California, Davis (2006).

23 C.-S. Yoo, B. Maddox, and V. Iota, MRS Proceedings 1104 (2008).

24 M. Krisch, D. L. Faber, R. Xu, D. Antonangeli, C. M. Aracne, A. Beraud, T.-C. Chiang, J. Zarestky, D. Y. Kim, E. I. Isaev, R. Ahuja, and B. Johansson, Proc. Natl. Acad. Sci. USA 108, 9342 (2011).

25 I. Loa, E. I. Isaev, M. I. McMahon, D. Y. Kim, B. Johansson, A. Bosak, and M. Krisch, Phys. Rev. Lett. 108, 045502 (2012).

26 R. Ramirez and L. Falicov, Phys. Rev. B 3, 2425 (1971).

27 J. Allen and R. Martin, Phys. Rev. Lett. 49, 1106 (1982).

28 G. K. Samudrala, G. M. Tsoi, and Y. K. Vohra, J. Phys.: Condens. Matter 24, 362201 (2012).

29 Y. Akahama, H. Fujihisa, and H. Kawamura, Phys. Rev. Lett. 94, 195503 (2005).

30 S. Louie and M. Cohen, Phys. Rev. B 10, 3237 (1974).

31 H. Luo, R. Greene, K. Ghandehari, T. Li, and A. Ruoff, Phys. Rev. B 50, 16232 (1994).

32 A. Lazicki, B. Maddox, W. Evans, C.-S. Yoo, A. McMahan, W. Pickett, R. Scalettar, M. Hu, and P. Chow, Phys. Rev. Lett. 95, 165503 (2005).

33 W. Xiao, D. Tan, X. Xiong, J. Liu, and J. Xu, PNAS 107, 14026 (2010).

34 J. Duthie and D. Pettifor, Phys. Rev. Lett. 38, 564 (1977).

35 M. MacPherson, G. Everett, D. Wohlleben, and M. Maple, Phys. Rev. Lett. 26, 20 (1971).

36 Z. Yin and W. Pickett, Phys. Rev. B 74, 205106 (2006).

37 Material Preparation Center, Ames Lab, US DOE, Ames, Iowa [http://www.mpc.ameslab.gov].

38 A. D. Chijioke, W. J. Nellis, A. Soldatov, and I. F. Silvera, J. Appl. Phys. 98, 114905 (2005).

${ }^{39}$ K. Shimizu, K. Amaya, and N. Suzuki, J. Phys. Soc. Jpn. 74, 1345 (2005).

40 M. Rivers, V. B. Prakapenka, A. Kubo, C. Pullins, C. M. Holl, and S. D. Jacobsen, High Press. Res. 28, 273 (2008).

41 M. Newville, J. of Synch. Rad. 12, 537 (2001).

42 B. Ravel and M. Newville, J. of Synch. Rad. 12, 537 (2005).

43 S. Zabinsky, J. J. Rehr, A. Ankudinov, R. C. Albers, and 
M. J. Eller, Phys. Rev. B 52, 2995 (1995).

44 F. H. Spedding, A. H. Daane, and K. W. Herrmann, Acta Crystallogr. 9, 559 (1956).

45 M. B. Maple, Appl. Phys. 9, 179 (1976).

${ }^{46}$ M. Maple, J. Wittig, and K. Kim, Phys. Rev. Lett. 23, 1375 (1969).

47 M. Zuckermann, Phys. Rev. 168, 390 (1968).

48 E. Müller-Hartmann and J. Zittartz, Z. Physik 234, 58 (1970).

49 E. Müller-Hartmann and J. Zittartz, Phys. Rev. Lett. 26, 428 (1971).

${ }^{50}$ W. Gey and E. Umlauf, Z. Physik 243, 241 (1971).

51 M. Maple, W. Fertig, A. Mota, L. DeLong, D. Wohlleben, and R. Fitzgerald, Solid State Commun. 11, 829 (1972).

52 J. Hamlin, V. Tissen, and J. Schilling, Physica C 451, 82 (2007).
53 J. Wittig, Phys. Rev. Lett. 46, 1431 (1981).

54 H. R. Mao, R. M. Hazen, P. M. Bell, and J. Wittig, J. Appl. Phys. 52, 4572 (1981).

55 J. Wittig, Valencies Instabilities, edited by P. Watcher and H. Boppart (North-Holland, Amsterdam, 1982) p. 427.

56 J. Roehler and R. Luebbers, Physica B 206-7, 368 (1995).

57 J. Bradley, K. Moore, M. Lipp, B. Mattern, J. Pacold, G. Seidler, P. Chow, E. Rod, Y. Xiao, and W. Evans, Phys. Rev. B 85, 100102 (2012).

58 K. Döbrich, G. Bihlmayer, K. Starke, J. Prieto, K. Rossnagel, H. Koh, E. Rotenberg, S. Blügel, and G. Kaindl, Phys. Rev. B 76, 035123 (2007).

${ }^{59}$ K. Jouda, S. Tanaka, and O. Aita, J. Phys.: Condens. Matter 9, 10789 (1997).

60 J. Lim, G. Fabbris, and J. S. Schilling (unpublished).

61 J. S. Schilling, Mat. Res. Soc. Symp. Proc. 22, 51 (1984). 